\title{
Comparação de métodos quantitativos etnobotânicos no estudo de plantas medicinais no semiárido da Paraíba, Nordeste do Brasil
}

\section{Guilherme Muniz Nunes ${ }^{1}, *$, Ezequiel da Costa Ferreira ${ }^{1}$, André dos Santos Souza ${ }^{1}$, Suellen da Silva Santos ${ }^{1}$, Camilla Marques de Lucena ${ }^{2}, * *$ e Reinaldo Farias Paiva de Lucena $^{1}$,***}

\begin{abstract}
${ }^{1}$ Universidade Federal da Paraíba. Centro de Ciências Exatas e da Natureza. Programa de Pós-Graduação em Desenvolvimento e Meio Ambiente - PRODEMA. João Pessoa-PB, Brasil (CEP 58051-900). *E-mail: guilhermemnunes@hotmail.com. ${ }^{2}$ Universidade Federal do Mato Grosso do Sul. Instituto de Biociências. Laboratório de Etnobotânica. Campo Grande-MS, Brasil (CEP 79070-900). **E-mail: camilla.lucena@ufms.br; ***E-mail: reinaldo.lucena@ufms.br.
\end{abstract}

Resumo. 0 uso de plantas no tratamento de enfermidades é historicamente antigo entre as populações humanas. A etnobotânica, como campo de conhecimento tem buscado compreender entre outros temas, a dinâmica do uso de plantas medicinais nas mais diversas populações humanas, para isso, diversas hipóteses e métodos quantitativos próprios têm sido desenvolvidos para analisar o conhecimento tradicional. 0 objetivo do presente estudo foi de avaliar e comparar o conhecimento sobre plantas medicinais por meio dos índices de importância relativa, valor de uso e frequência relativa de citação. Foram feitas entrevistas semiestruturadas em comunidades rurais de oito municípios de diferentes regiões incluídas no semiárido paraibano. Por meio das entrevistas foram identificadas 52 espécies de uso medicinal, com destaque para as famílias Fabaceae e Euphorbiaceae no número de espécies utilizadas. Foi observada uma correspondência entre as principais espécies amostradas nas listagens dos métodos aplicados, com destaque, principalmente para Myracroduon urundeuva e Sideroxylum obtusifolium. Os resultados obtidos sugerem uma necessidade de estabelecer estratégias de uso sustentável de modo a evitar pressões de uso sobre as espécies mais conhecidas e utilizadas.

Palavras-chave: Conhecimento tradicional; Etnobotânica quantitativa; Medicina tradicional; Caatinga.

Abstract. Comparison of ethnobotanical quantitative methods in the study of medicinal plants in the Semiarid Region of Paraíba, Northeast Brazil. The use of plants in the treatment of
Recebido

$19 / 10 / 2021$

Aceito

$03 / 01 / 2022$

Disponível on line

$31 / 01 / 2022$

Publicado

$30 / 04 / 2022$

Acesso aberto

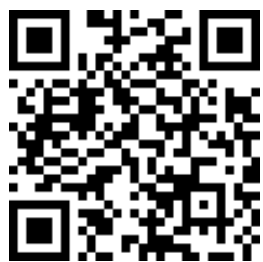

D) 0000-0003-2068-6436 Guilherme Muniz Nunes

ISSN 2359-1412/RBGAS-2021-0141/2022/9/21/3/37

Rev. Bras. Gest. Amb. Sustent.

http://revista.ecogestaobrasil.net 
diseases is historically ancient among human populations. Ethnobotany, as a Field of knowledge, has sought to understand, among other topics, the dynamics of the use of medicinal plants in the most diverse human populations, for that, several hypotheses and their own quantitative methods have been developed to analyze traditional knowledge. The objective of the present study was to evaluate and compare the knowledge about medicinal plants through the indices of relative importance, value of use and relative frequency of citation. Semi-structured interviews were carried out in rural communities in 8 municipalities in different regions included in the semi-arid region of Paraíba. Through the interviews, 52 species of medicinal use were identified, with emphasis on the Fabaceae and Euphorbiaceae families in the number of species used. Correspondence was observed between the main species sampled in the lists of the applied methods, especially for Myracroduon urundeuva and Sideroxylum obtusifolium. The results obtained suggest a need to establish sustainable use strategies in order to avoid pressures of use on the most known and used species.

Keywords: Traditional knowledge; Quantitative ethnobotany; Traditional medicine; Caatinga.

\author{
D) 0000-0003-4758-7171 \\ Ezequiel da Costa \\ Ferreira \\ (ㄷ) 0000-0001-8085-1881 \\ André dos Santos \\ Souza \\ (D) 0000-0002-9017-1457 \\ Suellen da Silva Santos \\ (1) 0000-0002-5126-8969 \\ Camilla Marques de \\ Lucena \\ (D) 0000-0002-1195-4315 \\ Reinaldo Farias Paiva \\ de Lucena
}

\section{Introdução}

O uso de plantas para cuidados com a saúde é tão antigo quanto o próprio ser humano, sendo os registros deste uso encontrado nas mais diversas civilizações, desde a antiguidade (Tomazzoni et al., 2006). Ao longo do tempo, a ciência têm evoluído na descoberta de fármacos para o tratamento de doenças, no entanto, nos países mais pobres a prática do uso de plantas medicinais e fitoterápicos ainda apresenta uma grande força, devido à falta de acesso e poder aquisitivo de parte da população para compra de medicamentos alopáticos, principalmente em áreas rurais (Bermúdez et al., 2005; Bhat et al., 2013; Vandebroek et al., 2011).

Deste modo, as populações carentes fazem uso de plantas medicinais para cuidados e tratamento de doenças, em diversas formas de preparos, como chás e lambedores, e esse conhecimento têm sido transmitido entre gerações (Dantas e Guimarães, 2007). No entanto, deve haver o cuidado de se fazer este uso de maneira sustentável, de modo a evitar que o uso indiscriminado leve à morte dos indivíduos vegetais utilizados, e consequente escassez do recurso, sendo necessário estudos conservacionistas para traçar estratégias de uso sustentável para as comunidades que utilizam esses recursos (Jha, 1995; Dhar et al., 2000; Gera et al., 2003).

Compreender as relações de grupos humanos com recursos vegetais, como o uso terapêutico das plantas, se propõe como principal objeto de estudo da etnobotânica, uma área de conhecimento interdisciplinar focada na compreensão das relações entre cultura e meio ambiente no uso de plantas (Oliveira et al., 2009). Cuidados com a saúde e conservação da biodiversidade estão entre os principais temas investigados e que têm recebido contribuições da etnobotânica (Vandebroek et al., 2011).

A Região Nordeste do Brasil é atualmente a que tem recebido o maior número de estudos etnobotânicos no país (Ritter et al., 2015). Estes estudos tem focado em diversas temáticas, como inventários de uso (Batista et al., 2017; Lucena et al., 2017), a diversidade de uso das plantas medicinais, e consenso sobre as informações fornecidas pelas pessoas 
(Coutinho et al., 2015; Santos et al., 2018), conhecimento sobre plantas alimentícias (Nunes et al., 2015; Nunes et al., 2018), prioridades de conservação de plantas úteis (Souza et al., 2017; Ribeiro et al., 2019), critérios de seleção de plantas úteis (Alencar et al., 2010; Guerra et al., 2015; Silva et al., 2018).

Diferentes hipóteses e métodos quantitativos têm sido propostos em etnobotânica para a investigação desses saberes locais, como a hipótese da versatilidade, que sugere que plantas medicinais exóticas são incorporadas à farmacopeias locais por meio da experimentação de plantas introduzidas para uso alimentício e ornamental, por exemplo (Bennett e Prance, 2000), essa hipótese é investigada pelo método da Importância Relativa (Almeida e Albuquerque, 2002; Bennett e Prance, 2000; Ferreira et al., 2021a). Outro exemplo é a Hipótese da Aparência Ecológica, testada pelo método do Valor de Uso, que propõe que no ambiente existem espécies aparentes, que apresentam maior porte e distribuição local, e espécies não aparentes, de menor porte e menor distribuição, esta hipótese considera que a espécies aparentes apresentam um maior uso (Phillips e Gentry, 1993a, 1993b; Ribeiro et al., 2014; Guerra et al., 2015).

Estudos etnobotânicos mais amplos, comparando distintas regiões em um determinado recorte geográfico e temporal, podem ser também úteis para elucidar características próprias de semelhanças e diferenças entre diferentes áreas e compreender a dinâmica do conhecimento e uso das plantas nestas áreas, buscando compreender as variáveis locais que possam justificar as semelhanças e diferenças no elenco de espécies utilizadas e suas aplicações (Bussmann et al., 2016; Ribeiro et al., 2017; Macedo et al., 2018; Ferreira 2021b).

Diante do exposto, o presente estudo parte da hipótese de que versatilidade, número de citações e número de informantes podem apresentar correspondência entre si no ranqueamento de plantas medicinais. 0 objetivo foi o de avaliar sob diferentes métodos quantitativos o uso e o conhecimento das espécies vegetais utilizadas com a finalidade medicinal, em comunidades rurais no estado da Paraíba, Nordeste do Brasil. Como objetivos específicos tem-se, a) Identificar as espécies vegetais medicinais conhecidas e utilizadas, e b) Analisar, ordenar e comparar as espécies vegetais para fins medicinais de acordo com sua Importância Relativa (IR), Valor de Uso (VU) e Frequência Relativa de Citação (FRC).

\section{Materiais e métodos}

\section{Área de estudo}

O estudo foi conduzido em oito municípios situados na Região Semiárida do Estado da Paraíba, e subdivididos entre as Mesorregiões do Agreste, Borborema e Sertão (Figura 1). A vegetação é arbustivo-arbórea, típica do ecossistema Caatinga (Velloso et al., 2002).

Solânea - Localizada na Mesorregião do Agreste e na Microrregião do Curimataú do Semiárido do Estado da Paraíba. 0 estudo foi realizado na Comunidade Capivara (Soares et al., 2013). É o município mais populoso desta amostragem, com 26.963 habitantes, território de 232,970 km² e 593 m de altitude (IBGE, 2010), é circundada pelos Municípios de Arara, Bananeiras, Borborema, Serraria e Pirpirituba, e dista $149 \mathrm{~km}$ da capital do estado.

Remígio - Localiza-se na Mesorregião do Agreste e Microrregião do Curimataú Oriental, a $593 \mathrm{~m}$ do nível do mar. 0 estudo foi realizado na Comunidade Rural de Coelho, a cerca de $7 \mathrm{~km}$ da sede do município (Coutinho et al., 2015). Abriga 17.581 habitantes e localiza-se a 157 km de João Pessoa, fazendo divisa com os Municípios de Areia, Arara, Esperança e Algodão de Jandaíra (IBGE,2010).

Soledade - Possui 13.739 habitantes e densidade populacional de $24,53 \mathrm{hab} / \mathrm{km}^{2}$ e altitude de $52 \mathrm{~m}$. Está situada a $186 \mathrm{~km}$ da capital do estado, João Pessoa, localizado na Microrregião do Curimataú Ocidental (IBGE, 2010). Tem como municípios circunvizinhos 
Olivedos, Pocinhos e Juazeirinho. 0 estudo foi realizado nas Comunidades Rurais de Barrocas e Cachoeira (Lucena et al., 2012).

Cabaceiras - Possui uma área de 452,925 km² e uma população estimada de 5.035 habitantes e altitude aproximada de 500 m (IBGE, 2010). Localiza-se na Mesorregião da Borborema e Microrregião do Cariri Ocidental, e dista $196 \mathrm{~km}$ da capital do estado, João Pessoa. Os municípios circunvizinhos são Campina Grande, Barra de São Miguel, São Domingos do Cariri, Boqueirão e São João do Cariri. Mais da metade da sua população vive na zona rural. 0 estudo foi conduzido na Comunidade São Francisco (Silva et al., 2014).

Congo - Localiza-se na Mesorregião da Borborema e Microrregião do Cariri Ocidental, no Semiárido do Estado da Paraíba. Sua população estimada é de 4.789 em um território de $333,471 \mathrm{~km}^{2}$ (IBGE, 2010). Situa-se a cerca de $212 \mathrm{~km}$ da capital do estado, João Pessoa, fazendo limite com Coxixola, Caraúbas, Camalaú e Sumé na Paraíba, e Santa Cruz do Capibaribe no Estado de Pernambuco. 0 estudo foi desenvolvido na Comunidade Rural de Santa Rita (Guerra et al., 2015).

São Mamede - 0 estudo foi conduzido na Comunidade de Várzea Alegre que se localiza a aproximadamente $6 \mathrm{~km}$ do centro urbano. São Mamede dista aproximadamente $278 \mathrm{~km}$ da capital do Estado, próximos dos Municípios de Santa Luzia, Quixaba e São José de Espinharas. Está localizado na Microrregião do Seridó Ocidental (Guerra et al., 2012). A população conta com 7.745 habitantes e densidade populacional de $14,60 \mathrm{hab} / \mathrm{km}^{2}$ e 263m no nível do mar (IBGE, 2010).

Lagoa - Localiza-se na Mesorregião do Sertão e Microrregião de Catolé do Rocha. Possui 4.681 habitantes distribuídos em 177,902 $\mathrm{km}^{2}$ e altitude de $480 \mathrm{~m}$ (IBGE, 2010). Está situado a cerca de $212 \mathrm{~km}$ da capital do estado, João Pessoa. Os estudos foram realizados na Comunidade Barroquinha, zona rural do município (Carvalho et al., 2012).

Itaporanga - 0 município localiza-se na Mesorregião do Sertão e Microrregião do Vale do Piancó. Apresenta população de cerca de 24.653 habitantes em uma área de $468,059 \mathrm{~m}^{2}$ e altitude estimada de $191 \mathrm{~m}$ (IBGE, 2010). A capital do estado fica a cerca de 426 km. Os municípios limítrofes são Boa Ventura, Diamante, Pedra Branca, São José de Caiana, Santana dos Garrotes e Piancó. A Comunidade Rural Pau d'Arco foi escolhida para a execução da pesquisa (Leite et al., 2012).
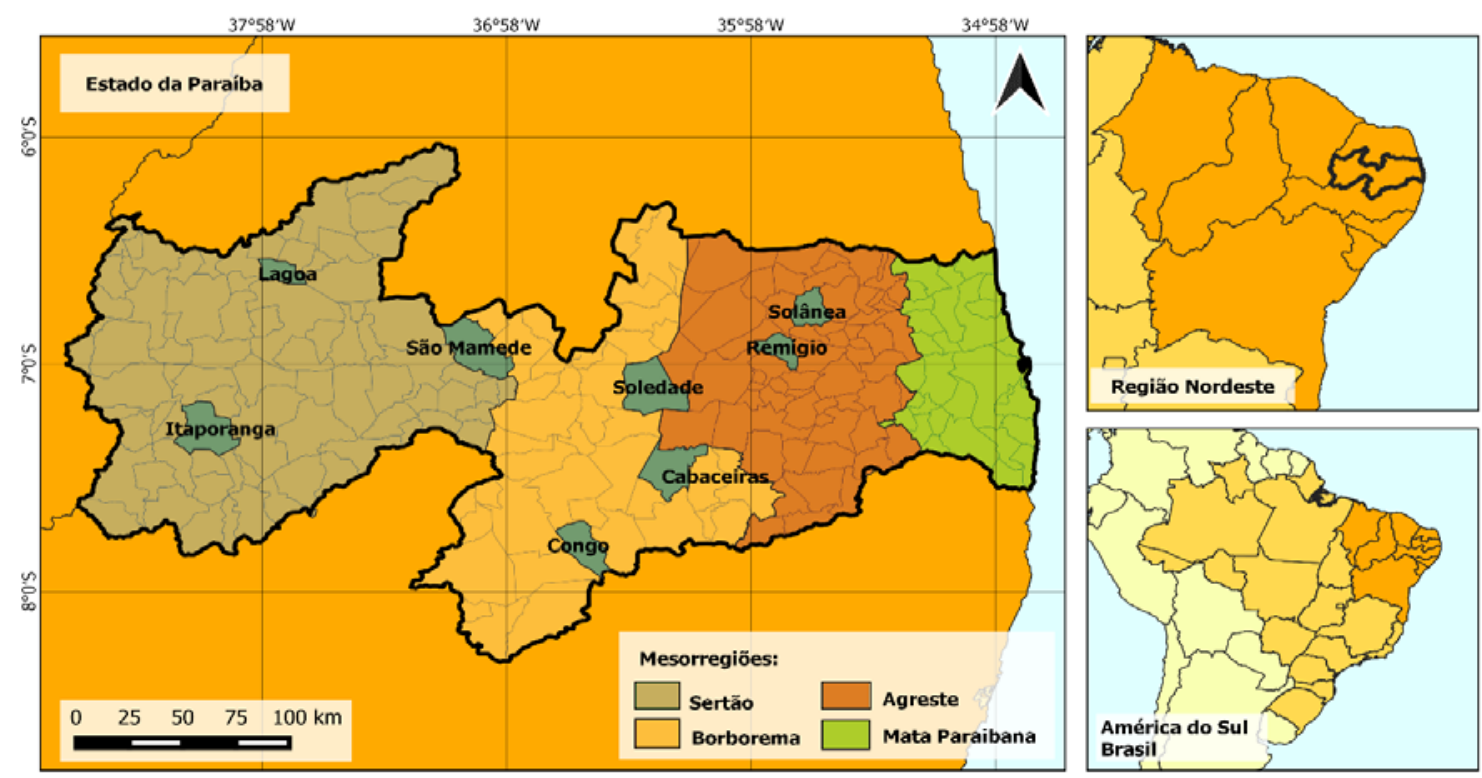

Figura 1. Localização dos municípios amostrados no estado da Paraíba, Nordeste do Brasil. Mapa: Ezequiel da Costa Ferreira (2018). 


\section{Inventário etnobotânico}

Foram conduzidas entrevistas semiestruturadas com os mantenedores dsd famílias (homem e mulher) (Albuquerque et al., 2010) em comunidades rurais de oito municípios do Estado da Paraíba, Nordeste, Brasil (Tabela 1). 0 método de entrevista semiestruturada consiste no estabelecimento de questões prévias pelo pesquisador antes da condução da entrevista, entretanto, neste método, as perguntas permitem flexibilidade no sentido de dar uma maior atenção a questões que possam surgir durante a entrevista (Albuquerque et al., 2010).

As entrevistas abordaram questões pertinentes às plantas lenhosas da vegetação nativa conhecidas e utilizadas para finalidades medicinais, a parte utilizada da planta, o modo de uso e a indicação terapêutica. Posteriormente, as indicações terapêuticas foram classificadas em categorias de sistemas corporais, a partir de uma adaptação da Classificação Internacional de Doenças (CID) da Organização Mundial de Saúde (OMS) (ICD, 2010).

As espécies citadas foram coletadas em áreas de vegetação próximas às comunidades com a colaboração de informantes chave por meio da técnica de turnê guiada, que consiste em percorrer a área de vegetação local acompanhado por um membro da comunidade reconhecido especialista local, isto é, uma pessoa de amplo conhecimento sobre a flora local. Durante a caminhada, o informante indica as espécies pelo nome vernacular para ser coletada e ter o nome científico validado (Albuquerque et al., 2010). As espécies foram herborizadas em campo, identificadas e incorporadas à coleção do herbário Jaime Coelho de Morais (EAN) do Centro de Ciências Agrárias (CCA) da Universidade Federal da Paraíba (UFPB).

Tabela 1. Número de pessoas entrevistadas por gênero, município e comunidade rural estudada no estado da Paraíba, Nordeste, Brasil.

\begin{tabular}{|l|c|c|c|c|}
\hline Município & Comunidade & Homens & Mulheres & Total \\
\hline Solânea & Capivara & 53 & 59 & 112 \\
\hline Remígio & Coelho & 19 & 23 & 42 \\
\hline Soledade & Barrocas & 8 & 7 & 15 \\
\hline Soledade & Cachoeira & 14 & 15 & 29 \\
\hline Cabaceiras & São Francisco & 53 & 70 & 123 \\
\hline Congo & Santa Rita & 41 & 57 & 98 \\
\hline São Mamede & Várzea Alegre & 18 & 19 & 37 \\
\hline Lagoa & Barroquinha & 25 & 41 & 66 \\
\hline Itaporanga & Pau d'Arco & 8 & 7 & 15 \\
\hline Total & & $\mathbf{2 3 9}$ & $\mathbf{2 9 8}$ & $\mathbf{5 3 7}$ \\
\hline
\end{tabular}

\section{Análise de dados etnobotânicos}

As propriedades terapêuticas citadas para cada espécie foram agrupadas em sistemas corporais, conforme adaptação realizada a partir da Classificação Internacional de Doenças da Organização Mundial de Saúde (ICD, 2010).

A importância local das espécies foi avaliada por meio de três métodos quantitativos distintos: O Valor de Uso (VU) (Phillips e Gentry, 1993a, 1993b; Rossato et al., 1999), Importância Relativa (IR) (Bennett e Prance, 2000) e a Frequência Relativa de Citação (FRC) (Tardío e Pardo-de-Santayana, 2008).

O Valor de Uso é um método proposto para avaliar o uso local das espécies (Phillips e Gentry, 1993a, 1993b), seu cálculo foi realizado para a categoria medicinal conforme adaptação a partir da seguinte fórmula (Rossato et al., 1999): VU = $\sum \mathrm{Ui} / \mathrm{n}$, onde $\mathrm{Ui}=$ número de indicações dadas pelos informantes, $\mathrm{n}=$ número de informantes. 
A Importância Relativa é um método proposto para avaliar a versatilidade, isto é, a diversidade de usos mencionados para cada espécie (Bennett e Prance, 2000). 0 valor máximo obtido neste cálculo é igual a 2, e quão mais próximo de 2 for a IR da espécie, maior será sua versatilidade. 0 cálculo é realizado seguindo a fórmula: IR $=$ NSC/NP. Onde NSC $=$ Número de sistemas corporais e NP = Número de propriedades (Bennett e Prance, 2000).

O NSC é obtido a partir do seguinte cálculo: NSC = NSCE/NSCEV. Sendo NSCE = Número de sistemas corporais atribuídos à espécie e NSCEV = Número de sistemas corporais atribuídos à espécie mais versátil. Sendo considerada mais versátil a espécie que teve suas indicações agrupadas em um maior número de sistemas corporais.

O NP é obtido pelo seguinte cálculo: NP $=$ NPE/NPEV. Onde NPE = Número de propriedades atribuídas à espécie; $\mathrm{NP}=$ Número de propriedades atribuídas à espécie mais versátil. Neste caso, a espécie mais versátil é aquela que for indicada para um maior número de propriedades terapêuticas.

A Frequência Relativa de Citação é um método de quantitativo de importância cultural que considera o número de informantes que citam o uso de uma determinada espécie (Tardío e Pardo-de-Santayana, 2008), é calculada a partir da fórmula: FRC = FC/N. Onde $\mathrm{FC}=$ Frequência de citação (número de informantes que citaram o uso de determinada espécie) e $\mathrm{N}$ = número de participantes da pesquisa. Os resultados obtidos por este método variam de 0 (quando nenhum informante cita a espécie) a 1 (quando todos os informantes citam a espécie).

\section{Comitê de Ética}

O estudo foi desenvolvido com aprovação do Comitê de Ética em Pesquisa com Seres Humanos (CEP), do Hospital Lauro Wanderley, da Universidade Federal da Paraíba, com registro de protocolo CEP/HULW no 297/2011, Folha de Rosto no 420134.

\section{Resultados}

\section{Espécies identificadas}

Foram registradas e identificadas 52 plantas medicinais nas comunidades estudadas, das quais três foram identificadas apenas a nível de gênero. Outras nove plantas citadas não foram identificadas. As espécies identificadas estão distribuídas em 44 gêneros e 19 famílias. As famílias com maior número de espécies foram Fabaceae (14 spp.), Euphorbiaceae (8 spp.), Anacardiaceae e Bignoniaceae (ambas com 4 spp. cada). (Tabela 2).

Tabela 2. Espécies de plantas medicinais utilizadas nas comunidades rurais estudadas nos Municípios de Solânea, Remígio, Soledade, Cabaceiras, Congo, São Mamede, Lagoa e Itaporanga, no Semiárido paraibano, Nordeste do Brasil. IR = Importância Relativa; VU = Valor de Uso; FRC = Frequência Relativa de Citação.

\begin{tabular}{|l|l|c|c|c|}
\hline $\begin{array}{l}\text { Família/Nome científico } \\
\text { (Nome vernacular) }\end{array}$ & \multicolumn{1}{|c|}{ Município } & IR & VU & FRC \\
\hline Anacardiaceae & & & & \\
\hline \multirow{4}{*}{$\begin{array}{l}\text { Myracrodruon urundeuva } \\
\text { Allemão (aroeira) }\end{array}$} & Solânea & 2,00 & 1,27 & 0,79 \\
\cline { 2 - 5 } & Remígio & 1,28 & 0,79 & 0,55 \\
\cline { 2 - 5 } & Soledade - Barrocas & 1,56 & 0,48 & 0,47 \\
\cline { 2 - 5 } & Soledade - Cachoeira & 1,51 & 0,67 & 0,48 \\
\cline { 2 - 5 } & Cabaceiras & 1,87 & 0,77 & 0,59 \\
\cline { 2 - 5 } & Congo & 1,49 & 0,65 & 0,43 \\
\hline
\end{tabular}


Tabela 2. Continuação.

\begin{tabular}{|c|c|c|c|c|}
\hline $\begin{array}{l}\text { Família/Nome científico } \\
\text { (Nome vernacular) }\end{array}$ & Município & IR & VU & FRC \\
\hline \multirow{3}{*}{$\begin{array}{l}\text { Myracrodruon urundeuva } \\
\text { Allemão (aroeira) }\end{array}$} & São Mamede & 1,75 & 0,67 & 0,27 \\
\hline & Lagoa & 1,70 & 0,77 & 0,47 \\
\hline & Itaporanga & 2,00 & 0,47 & 0,33 \\
\hline \multirow{5}{*}{$\begin{array}{l}\text { Schinopsis brasiliensis Engl. } \\
\text { (baraúna) }\end{array}$} & Solânea & 0,91 & 0,20 & 0,13 \\
\hline & Remígio & 0,43 & 0,05 & 0,02 \\
\hline & Soledade - Cachoeira & 0,61 & 0,21 & 0,10 \\
\hline & Cabaceiras & 0,46 & 0,02 & 0,02 \\
\hline & Congo & 0,52 & 0,11 & 0,08 \\
\hline Spondias sp. (cajazeira) & Lagoa & 0,19 & 0,02 & 0,02 \\
\hline \multirow{5}{*}{$\begin{array}{l}\text { Spondias tuberosa Arruda } \\
\text { (umbuzeiro) }\end{array}$} & Solânea & 0,21 & 0,03 & 0,02 \\
\hline & Soledade - Barrocas & 0,52 & 0,10 & 0,07 \\
\hline & Cabaceiras & 0,94 & 0,16 & 0,15 \\
\hline & Congo & 0,40 & 0,06 & 0,05 \\
\hline & São Mamede & 0,22 & 0,03 & 0,03 \\
\hline \multicolumn{5}{|l|}{ Apocynaceae } \\
\hline \multirow{4}{*}{$\begin{array}{l}\text { Aspidosperma pyrifolium Mart. \& } \\
\text { Zucc. (pereiro) }\end{array}$} & Remígio & 0,21 & 0,02 & 0,02 \\
\hline & Congo & 0,55 & 0,04 & 0,04 \\
\hline & São Mamede & 0,45 & 0,08 & 0,08 \\
\hline & Lagoa & 0,45 & 0,06 & 0,03 \\
\hline \multicolumn{5}{|l|}{ Arecaceae } \\
\hline \multirow{3}{*}{$\begin{array}{l}\text { Syagrus oleracea (Mart.) Becc } \\
\text { (côco catolé) }\end{array}$} & Cabaceiras & 0,46 & 0,02 & 0,02 \\
\hline & Congo & 0,66 & 0,09 & 0,08 \\
\hline & Lagoa & 0,56 & 0,47 & 0,05 \\
\hline \multicolumn{5}{|l|}{ Bignoniaceae } \\
\hline \multirow{2}{*}{$\begin{array}{l}\text { Jocasanda momosifolia (Mart ex. } \\
\text { DC) Standl. (Caroba) }\end{array}$} & Lagoa & 0,37 & 0,03 & 0,03 \\
\hline & Itaporanga & 1,34 & 0,20 & 0,13 \\
\hline \multirow{3}{*}{ Handroanthus sp. (pau d'arco) } & Cabaceiras & 0,77 & 0,04 & 0,04 \\
\hline & Congo & 1,08 & 0,17 & 0,07 \\
\hline & São Mamede & 0,68 & 0,19 & 0,08 \\
\hline \multirow{2}{*}{$\begin{array}{l}\text { Handroanthus impetiginosa } \\
\text { (Mart ex. DC) Standl (pau d'arco } \\
\text { roxo) }\end{array}$} & Solânea & 0,83 & 0,09 & 0,08 \\
\hline & Congo & 0,41 & 0,20 & 0,02 \\
\hline $\begin{array}{l}\text { Tabebuia aurea (Silva Manso) } \\
\text { Benth. \& Hook.f. ex S. Moore } \\
\text { (craibeira) }\end{array}$ & Congo & 0,14 & 0,01 & 0,01 \\
\hline \multicolumn{5}{|l|}{ Burseraceae } \\
\hline \multirow{9}{*}{$\begin{array}{l}\text { Commiphora leptophloeos (Mart.) } \\
\text { J.B. Gillett (umburana) }\end{array}$} & Solânea & 0,99 & 0,27 & 0,20 \\
\hline & Remígio & 0,21 & 0,05 & 0,05 \\
\hline & Soledade - Barrocas & 0,35 & 0,10 & 0,07 \\
\hline & Soledade - Cachoeira & 0,37 & 0,13 & 0,10 \\
\hline & Cabaceiras & 1,50 & 0,37 & 0,33 \\
\hline & Congo & 0,73 & 0,14 & 0,12 \\
\hline & São Mamede & 0,53 & 0,11 & 0,11 \\
\hline & Lagoa & 1,13 & 0,14 & 0,09 \\
\hline & Itaporanga & 1,34 & 0,27 & 0,27 \\
\hline \multicolumn{5}{|l|}{ Capparaceae } \\
\hline $\begin{array}{l}\text { Capparis jacobinae Moric. ex } \\
\text { Eichlee (icó) }\end{array}$ & Remígio & 0,63 & 0,07 & 0,02 \\
\hline Crateava trapia L. (trapiá) & Lagoa & 0,26 & 0,06 & 0,06 \\
\hline \multirow{2}{*}{$\begin{array}{l}\text { Cynophalla flexuosa (L.) J.Presl } \\
\text { (feijão brabo) }\end{array}$} & Solânea & 0,51 & 0,04 & 0,03 \\
\hline & Remígio & 0,21 & 0,02 & 0,02 \\
\hline
\end{tabular}


Tabela 2. Continuação.

\begin{tabular}{|c|c|c|c|c|}
\hline $\begin{array}{l}\text { Família/Nome científico } \\
\text { (Nome vernacular) }\end{array}$ & Município & IR & VU & FRC \\
\hline \multicolumn{5}{|l|}{ Celastraceae } \\
\hline \multirow{7}{*}{$\begin{array}{l}\text { Monteverdia rigida (Mart.) Biral } \\
\text { (bom nome) }\end{array}$} & Solânea & 1,46 & 0,45 & 0,32 \\
\hline & Remígio & 0,63 & 0,17 & 0,12 \\
\hline & Soledade - Barrocas & 1,39 & 0,57 & 0,40 \\
\hline & Soledade - Cachoeira & 2,00 & 0,96 & 0,48 \\
\hline & Cabaceiras & 1,07 & 0,08 & 0,08 \\
\hline & Congo & 0,76 & 0,14 & 0,12 \\
\hline & Lagoa & 0,56 & 0,05 & 0,03 \\
\hline \multicolumn{5}{|l|}{ Chrysobalanaceae } \\
\hline \multirow{2}{*}{ Licania rigida Benth. (oiticica) } & São Mamede & 0,31 & 0,06 & 0,05 \\
\hline & Lagoa & 0,26 & 0,09 & 0,09 \\
\hline \multicolumn{5}{|l|}{ Combretaceae } \\
\hline \multirow{4}{*}{$\begin{array}{l}\text { Combretum fruticosum (Loefl) } \\
\text { Stuntz (mufumbo) }\end{array}$} & Congo & 0,70 & 0,21 & 0,11 \\
\hline & São Mamede & 1,29 & 0,39 & 0,24 \\
\hline & Lagoa & 0,98 & 0,68 & 0,42 \\
\hline & Itaporanga & 1,34 & 0,33 & 0,33 \\
\hline $\begin{array}{l}\text { Thiloa glaucocarpa (Mart.) } \\
\text { Eichler (joão mole) }\end{array}$ & Solânea & 0,17 & 0,01 & 0,01 \\
\hline \multicolumn{5}{|l|}{ Euphorbiaceae } \\
\hline \multirow{8}{*}{$\begin{array}{l}\text { Cnidoscolus quercifolius Pohl. } \\
\text { (favela) }\end{array}$} & Solânea & 0,37 & 0,04 & 0,04 \\
\hline & Remígio & 0,50 & 0,12 & 0,07 \\
\hline & Soledade - Barrocas & 1,74 & 0,57 & 0,53 \\
\hline & Soledade - Cachoeira & 1,57 & 1,42 & 0,79 \\
\hline & Cabaceiras & 1,20 & 0,12 & 0,11 \\
\hline & Congo & 1,17 & 0,29 & 0,15 \\
\hline & São Mamede & 1,83 & 1,33 & 0,70 \\
\hline & Itaporanga & 0,66 & 0,07 & 0,07 \\
\hline \multirow{9}{*}{$\begin{array}{l}\text { Croton blanchetianus Baill. } \\
\text { (Marmeleiro) }\end{array}$} & Solânea & 0,50 & 0,57 & 0,46 \\
\hline & Remígio & 0,21 & 0,21 & 0,17 \\
\hline & Soledade - Barrocas & 0,44 & 0,19 & 0,20 \\
\hline & Soledade - Cachoeira & 0,18 & 0,29 & 0,24 \\
\hline & Cabaceiras & 0,83 & 0,32 & 0,28 \\
\hline & Congo & 0,27 & 0,12 & 0,11 \\
\hline & São Mamede & 0,22 & 0,33 & 0,30 \\
\hline & Lagoa & 0,87 & 0,73 & 0,53 \\
\hline & Itaporanga & 0,66 & 0,14 & 0,13 \\
\hline \multirow{2}{*}{$\begin{array}{l}\text { Croton heliotropiifolius } \\
\text { Kunth.(quebra faca) }\end{array}$} & Soledade - Barrocas & 0,52 & 0,10 & 0,13 \\
\hline & Soledade - Cachoeira & 0,74 & 0,25 & 0,14 \\
\hline \multirow{5}{*}{$\begin{array}{l}\text { Croton rhamnifolius Kunt. } \\
\text { (velame) }\end{array}$} & Solânea & 0,33 & 0,02 & 0,02 \\
\hline & Remígio & 0,43 & 0,05 & 0,02 \\
\hline & Congo & 0,45 & 0,06 & 0,04 \\
\hline & São Mamede & 0,22 & 0,08 & 0,08 \\
\hline & Lagoa & 0,87 & 0,08 & 0,03 \\
\hline \multirow{2}{*}{$\begin{array}{l}\text { Croton sincorensis Mart. } \\
\text { (marmeleiro branco) }\end{array}$} & Soledade - Barrocas & 0,77 & 0,14 & 0,13 \\
\hline & Soledade - Cachoeira & 0,18 & 0,04 & 0,03 \\
\hline \multirow{6}{*}{$\begin{array}{l}\text { Jatropha mollissima (Pohl) Baill. } \\
\text { (pinhão brabo) }\end{array}$} & Solânea & 0,66 & 0,12 & 0,09 \\
\hline & Remígio & 0,28 & 0,12 & 0,07 \\
\hline & Soledade - Barrocas & 1,03 & 0,38 & 0,40 \\
\hline & Soledade - Cachoeira & 0,43 & 0,42 & 0,31 \\
\hline & Cabaceiras & 1,36 & 0,50 & 0,31 \\
\hline & Congo & 0,49 & 0,30 & 0,17 \\
\hline
\end{tabular}


Tabela 2. Continuação.

\begin{tabular}{|c|c|c|c|c|}
\hline $\begin{array}{l}\text { Família/Nome científico } \\
\text { (Nome vernacular) }\end{array}$ & Município & IR & VU & FRC \\
\hline \multirow{2}{*}{$\begin{array}{l}\text { Jatropha mollissima (Pohl) Baill. } \\
\text { (pinhão brabo) }\end{array}$} & São Mamede & 0,88 & 0,44 & 0,32 \\
\hline & Lagoa & 0,26 & 0,06 & 0,05 \\
\hline \multirow{2}{*}{$\begin{array}{l}\text { Jatroph aribifolia (Pohl) Baill. } \\
\text { (pinhão manso) }\end{array}$} & Solânea & 0,66 & 0,03 & 0,04 \\
\hline & Congo & 0,14 & 0,01 & 0,01 \\
\hline $\begin{array}{l}\text { Sapium glandulosum (L.) Morong } \\
\text { (burra leiiteira) }\end{array}$ & Soledade - Cachoeira & 0,36 & 0,07 & 0,07 \\
\hline \multicolumn{5}{|l|}{ Fabaceae } \\
\hline \multirow{9}{*}{$\begin{array}{l}\text { Amburana cearensis (Allemão) A. } \\
\text { C. Sm. (cumaru) }\end{array}$} & Solânea & 0,94 & 1,07 & 0,67 \\
\hline & Remígio & 1,35 & 0,62 & 0,33 \\
\hline & Soledade - Barrocas & 1,48 & 0,62 & 0,47 \\
\hline & Soledade - Cachoeira & 1,71 & 1,17 & 0,59 \\
\hline & \begin{tabular}{|l|} 
Cabaceiras \\
\end{tabular} & 0,23 & 0,05 & 0,03 \\
\hline & Congo & 0,70 & 0,18 & 0,15 \\
\hline & São Mamede & 0,85 & 0,14 & 0,08 \\
\hline & Lagoa & 0,68 & 0,67 & 0,47 \\
\hline & Itaporanga & 0,66 & 0,13 & 0,13 \\
\hline \multirow{9}{*}{$\begin{array}{l}\text { Anadenanthera colubrina (Vell.) } \\
\text { Brenan (angico) }\end{array}$} & Solânea & 1,19 & 0,39 & 0,28 \\
\hline & Remígio & 0,21 & 0,05 & 0,02 \\
\hline & Soledade - Barrocas & 0,88 & 0,29 & 0,27 \\
\hline & Soledade - Cachoeira & 0,37 & 0,08 & 0,07 \\
\hline & \begin{tabular}{|l|} 
Cabaceiras \\
\end{tabular} & 0,77 & 0,14 & 0,11 \\
\hline & Congo & 0,97 & 0,40 & 0,30 \\
\hline & São Mamede & 0,90 & 0,17 & 0,11 \\
\hline & Lagoa & 0,64 & 0,30 & 0,26 \\
\hline & Itaporanga & 0,66 & 0,07 & 0,07 \\
\hline \multirow{8}{*}{$\begin{array}{l}\text { Bauhinia cheilantha (Bong.) } \\
\text { Steud. (mororó) }\end{array}$} & Solânea & 0,37 & 0,06 & 0,05 \\
\hline & Remígio & 0,92 & 0,12 & 0,05 \\
\hline & Soledade - Barrocas & 1,73 & 0,57 & 0,47 \\
\hline & Soledade - Cachoeira & 1,94 & 1,08 & 0,59 \\
\hline & Cabaceiras & 1,53 & 0,11 & 0,08 \\
\hline & Congo & 0,55 & 0,09 & 0,07 \\
\hline & Lagoa & 0,64 & 0,06 & 0,05 \\
\hline & Itaporanga & 0,66 & 0,07 & 0,07 \\
\hline $\begin{array}{l}\text { Bauhinia pentandra (Bong) Vogel } \\
\text { ex Steud. (mororó) }\end{array}$ & São Mamede & 0,99 & 0,28 & 0,22 \\
\hline \multirow{6}{*}{$\begin{array}{l}\text { Erythrina velutina Willd. } \\
\text { (mulungu) }\end{array}$} & Solânea & 0,66 & 0,07 & 0,05 \\
\hline & \begin{tabular}{|l|} 
Remígio \\
\end{tabular} & 0,50 & 0,12 & 0,12 \\
\hline & Soledade - Barrocas & 1,05 & 0,29 & 0,27 \\
\hline & Soledade - Cachoeira & 0,85 & 0,25 & 0,14 \\
\hline & \begin{tabular}{|l|} 
Cabaceiras \\
\end{tabular} & 0,83 & 0,10 & 0,07 \\
\hline & Congo & 0,14 & 0,02 & 0,02 \\
\hline \multirow{5}{*}{ Hymenaea courbaril L. (jatobá) } & \begin{tabular}{|l|} 
Solânea \\
\end{tabular} & 0,58 & 0,08 & 0,06 \\
\hline & Remígio & 0,92 & 0,36 & 0,24 \\
\hline & Cabaceiras & 0,46 & 0,11 & 0,10 \\
\hline & Congo & 0,45 & 0,13 & 0,10 \\
\hline & Lagoa & 0,82 & 0,18 & 0,14 \\
\hline \multirow{2}{*}{ Inga sp. (ingá) } & Cabaceiras & 0,23 & 0,01 & 0,01 \\
\hline & Congo & 0,14 & 0,01 & 0,01 \\
\hline \multirow{3}{*}{$\begin{array}{l}\text { Libidibia ferrea (Mart. ex Tul.) } \\
\text { L.P. Queiroz (jucá) }\end{array}$} & Solânea & 0,58 & 0,13 & 0,11 \\
\hline & Remígio & 0,63 & 0,10 & 0,07 \\
\hline & Soledade - Barrocas & 1,12 & 0,33 & 0,27 \\
\hline
\end{tabular}


Tabela 2. Continuação.

\begin{tabular}{|c|c|c|c|c|}
\hline $\begin{array}{l}\text { Família/Nome científico } \\
\text { (Nome vernacular) }\end{array}$ & Município & IR & VU & FRC \\
\hline \multirow{5}{*}{$\begin{array}{l}\text { Libidibia ferrea (Mart. ex Tul.) } \\
\text { L.P. Queiroz (jucá) }\end{array}$} & Soledade - Cachoeira & 0,91 & 0,38 & 0,21 \\
\hline & Cabaceiras & 1,16 & 0,10 & 0,10 \\
\hline & \begin{tabular}{|l|} 
Congo \\
\end{tabular} & 0,59 & 0,08 & 0,08 \\
\hline & \begin{tabular}{|l|} 
São Mamede \\
\end{tabular} & 1,85 & 0,78 & 0,22 \\
\hline & Lagoa & 1,28 & 0,18 & 0,12 \\
\hline $\begin{array}{l}\text { Mimosa ophthalmocentra Marth. } \\
\text { ex Benth. (jurema de imbira) }\end{array}$ & Soledade - Barrocas & 0,26 & 0,05 & 0,07 \\
\hline \multirow{7}{*}{$\begin{array}{l}\text { Mimosa tenuiflora (Willd.) Poir. } \\
\text { (jurema preta) }\end{array}$} & Solânea & 0,50 & 0,02 & 0,03 \\
\hline & Remígio & 0,63 & 0,10 & 0,05 \\
\hline & Soledade - Cachoeira & 0,61 & 0,21 & 0,14 \\
\hline & Cabaceiras & 1,00 & 0,08 & 0,07 \\
\hline & Congo & 0,83 & 0,47 & 0,34 \\
\hline & São Mamede & 1,43 & 0,67 & 0,35 \\
\hline & Lagoa & 1,44 & 0,86 & 0,56 \\
\hline \multirow{5}{*}{$\begin{array}{l}\text { Piptadenia stipulacea (Benth.) } \\
\text { Ducke (jurema branca) }\end{array}$} & Soledade - Cachoeira & 0,18 & 0,04 & 0,03 \\
\hline & Cabaceiras & 0,30 & 0,02 & 0,02 \\
\hline & \begin{tabular}{|l|} 
Congo \\
\end{tabular} & 0,14 & 0,02 & 0,02 \\
\hline & São Mamede & 0,22 & 0,28 & 0,03 \\
\hline & Lagoa & 0,37 & 0,03 & 0,03 \\
\hline $\begin{array}{l}\text { Pitchecellobium diversifolium } \\
\text { Benth (espinheiro) }\end{array}$ & São Mamede & 0,22 & 0,03 & 0,03 \\
\hline \multirow{9}{*}{$\begin{array}{l}\text { Cenostigma pyramidale (Tul.) } \\
\text { Gagnon \& G. P. Lewis } \\
\text { (catingueira) }\end{array}$} & Solânea & 1,19 & 0,67 & 0,53 \\
\hline & \begin{tabular}{|l|} 
Remígio \\
\end{tabular} & 1,50 & 0,90 & 0,36 \\
\hline & Soledade - Barrocas & 0,95 & 0,53 & 0,47 \\
\hline & Soledade - Cachoeira & 0,85 & 0,58 & 0,31 \\
\hline & Cabaceiras & 1,30 & 0,59 & 0,45 \\
\hline & \begin{tabular}{|l|} 
Congo \\
\end{tabular} & 0,91 & 0,62 & 0,42 \\
\hline & \begin{tabular}{|l|} 
São Mamede \\
\end{tabular} & 0,53 & 0,36 & 0,22 \\
\hline & Lagoa & 0,98 & 0,24 & 0,12 \\
\hline & \begin{tabular}{|l|} 
Itaporanga \\
\end{tabular} & 1,34 & 0,73 & 0,47 \\
\hline $\begin{array}{l}\text { Senegalia polyphylla (D.C.) } \\
\text { Britton \& Rose (unha de gato) }\end{array}$ & Lagoa & 0,19 & 0,02 & 0,02 \\
\hline \multicolumn{5}{|l|}{ Malvaceae } \\
\hline \multirow{2}{*}{$\begin{array}{l}\text { Chorisia glaziovii (Kuntze) E. } \\
\text { Santos (barriguda) }\end{array}$} & Solânea & 0,54 & 0,06 & 0,06 \\
\hline & Soledade - Barrocas & 0,28 & 0,05 & 0,07 \\
\hline $\begin{array}{l}\text { Guazuma ulmifolia Lam. } \\
\text { (mutamba) }\end{array}$ & Lagoa & 0,19 & 0,02 & 0,02 \\
\hline \multirow{5}{*}{$\begin{array}{l}\text { Pseudobombax marginatum (A. } \\
\text { St.-Hil., Juss. \& Cambress.) A. } \\
\text { Robyns (imbiratã) }\end{array}$} & Soledade - Barrocas & 0,95 & 0,33 & 0,33 \\
\hline & Soledade - Cachoeira & 0,85 & 0,42 & 0,31 \\
\hline & Cabaceiras & 1,80 & 0,33 & 0,25 \\
\hline & \begin{tabular}{|l|} 
Congo \\
\end{tabular} & 0,72 & 0,09 & 0,07 \\
\hline & São Mamede & 0,76 & 0,14 & 0,08 \\
\hline \multicolumn{5}{|l|}{ Meliaceae } \\
\hline \multirow{3}{*}{ Cedrela odorata L. (cedro) } & Remígio & 0,43 & 0,05 & 0,02 \\
\hline & Congo & 0,14 & 0,01 & 0,01 \\
\hline & Lagoa & 0,19 & 0,02 & 0,02 \\
\hline \multicolumn{5}{|l|}{ Olacaceae } \\
\hline \multirow{4}{*}{ Ximenia americana L. (ameixa) } & Solânea & 1,19 & 0,34 & 0,23 \\
\hline & Remígio & 0,43 & 0,10 & 0,05 \\
\hline & Soledade - Barrocas & 1,83 & 0,86 & 0,67 \\
\hline & Soledade - Cachoeira & 1,51 & 1,42 & 0,72 \\
\hline
\end{tabular}


Tabela 2. Continuação.

\begin{tabular}{|c|c|c|c|c|}
\hline $\begin{array}{l}\text { Família/Nome científico } \\
\text { (Nome vernacular) }\end{array}$ & Município & IR & VU & FRC \\
\hline \multirow{5}{*}{ Ximenia americana L. (ameixa) } & Cabaceiras & 1,07 & 0,15 & 0,13 \\
\hline & Congo & 1,50 & 0,64 & 0,32 \\
\hline & São Mamede & 0,68 & 0,19 & 0,11 \\
\hline & Lagoa & 1,33 & 0,35 & 0,23 \\
\hline & \begin{tabular}{|l|} 
Itaporanga \\
\end{tabular} & 0,66 & 0,40 & 0,40 \\
\hline \multicolumn{5}{|l|}{ Plumbaginaceae } \\
\hline Plumbago scandens L. (louro) & Lagoa & 0,19 & 0,02 & 0,02 \\
\hline \multicolumn{5}{|l|}{ Poligonaceae } \\
\hline $\begin{array}{l}\text { Triplaris gardneriana Wedd. } \\
\text { cuaçu) }\end{array}$ & Lagoa & 0,37 & 0,03 & 0,03 \\
\hline \multicolumn{5}{|l|}{ Rhamnaceae } \\
\hline \multirow{9}{*}{ Ziziphus joazeiro Mart. (juazeiro) } & Solânea & 0,66 & 0,24 & 0,25 \\
\hline & Remígio & 0,77 & 0,24 & 0,21 \\
\hline & Soledade - Barrocas & 0,86 & 0,38 & 0,40 \\
\hline & Soledade - Cachoeira & 0,98 & 0,58 & 0,38 \\
\hline & Cabaceiras & 0,77 & 0,47 & 0,35 \\
\hline & Congo & 1,10 & 0,59 & 0,38 \\
\hline & São Mamede & 1,85 & 0,94 & 0,51 \\
\hline & Lagoa & 1,21 & 0,64 & 0,45 \\
\hline & \begin{tabular}{|l|} 
Itaporanga \\
\end{tabular} & 1,00 & 0,14 & 0,13 \\
\hline \multicolumn{5}{|l|}{ Rubiaceae } \\
\hline \multirow{2}{*}{$\begin{array}{l}\text { Coutarea hexandra (Jacq.) K. } \\
\text { Schum (quina quina) }\end{array}$} & Solânea & 0,17 & 0,01 & 0,01 \\
\hline & Lagoa & 0,53 & 0,02 & 0,06 \\
\hline $\begin{array}{l}\text { Machaonia spinosa Cham. \& } \\
\text { Schltdl. (quebra faca) }\end{array}$ & Lagoa & 0,37 & 0,03 & 0,03 \\
\hline \multirow{3}{*}{$\begin{array}{l}\text { Tocoyena formosa (Cham. \& } \\
\text { Schltdle) K. Schum (jenipapo } \\
\text { brabo) }\end{array}$} & Congo & 0,27 & 0,03 & 0,03 \\
\hline & Lagoa & 0,19 & 0,02 & 0,02 \\
\hline & \begin{tabular}{|l|} 
Itaporanga \\
\end{tabular} & 1,34 & 0,13 & 0,13 \\
\hline \multicolumn{5}{|l|}{ Sapotaceae } \\
\hline \multirow{8}{*}{$\begin{array}{l}\text { Sideroxylon obtusifolium (Roem. } \\
\text { \& Schult.) T.D. Penn. (quixabeira) }\end{array}$} & Solânea & 1,38 & 0,64 & 0,38 \\
\hline & \begin{tabular}{|l|} 
Remígio \\
\end{tabular} & 2,00 & 0,64 & 0,24 \\
\hline & Soledade - Barrocas & 1,65 & 0,71 & 0,73 \\
\hline & Soledade - Cachoeira & 1,57 & 1,04 & 0,66 \\
\hline & Cabaceiras & 2,00 & 0,96 & 0,74 \\
\hline & Congo & 2,00 & 1,41 & 0,76 \\
\hline & São Mamede & 1,85 & 0,50 & 0,22 \\
\hline & Lagoa & 2,00 & 1,09 & 0,59 \\
\hline \multicolumn{5}{|l|}{ Indeterminadas } \\
\hline \multirow{3}{*}{ Indet. 1 (jaramataia) } & Lagoa & 1,48 & 0,27 & 0,12 \\
\hline & Cabaceiras & 0,23 & 0,02 & 0,01 \\
\hline & Congo & 0,14 & 0,01 & 0,01 \\
\hline Indet. 2 (balço) & Lagoa & 1,02 & 0,14 & 0,12 \\
\hline Indet. 3 (pau gonçalo) & Lagoa & 0,37 & 0,05 & 0,03 \\
\hline Indet. 4 (amorosa preta) & Solânea & 0,17 & 0,01 & 0,01 \\
\hline Indet. 5 (mapirunga) & Solânea & 0,74 & 0,11 & 0,05 \\
\hline Indet. 6 (louro) & Remígio & 0,43 & 0,10 & 0,02 \\
\hline Indet. 7 (catinga branca) & Congo & 0,14 & 0,15 & 0,12 \\
\hline \multirow{2}{*}{ Indet. 8 (pau leite) } & Soledade - Cachoeira & 0,83 & 0,24 & 0,14 \\
\hline & Congo & 0,90 & 0,02 & 0,01 \\
\hline Indet. 9 (pau piranha) & Congo & 0,17 & 0,01 & 0,01 \\
\hline
\end{tabular}




\section{Comparação de métodos quantitativos nas áreas estudadas}

Mesorregião do Agreste (Solânea e Remígio). Um comparativo da colocação das principais espécies conforme cada método quantitativo aplicado nesta mesorregião é apresentado na Tabela 3. Nesta mesorregião, seis espécies obtiveram valor alto para a IR ( $\geq 1$ ) no Município de Solânea, sendo Myracrodruon urundeuva Allemão (aroeira, IR = 2), Monteverdia rigida (Mart.) Biral (bom nome, IR $=1,46$ ), Sideroxylon obtusifolium (Roem. \& Schult.) T.D. Penn. (quixabeira) (IR = 1,38), Anadenanthera colubrina (Vell.) Brenan (angico), Cenostigma pyramidale (Tul.) Gagnon \& G. P. Lewis (catingueira) e Ximenia americana L. (ameixa) (ambas com IR =1,19). Quanto ao Valor de Uso, M. urundeuva (VU = $1,27)$, C. pyramidale $(0,67)$ e $S$. obtusifolium $(0,64)$ também apresentaram os maiores valores, sendo uma exceção aqui Amburana cearensis (Allemão) A. C. Sm. (cumaru) (VU = 1,07), que obteve destaque no VU, mas não na IR. Para a FRC o destaque foi para $M$. urundeuva $(\mathrm{FRC}=0,79), A$. cearensis $(0,67)$ e $C$. pyramidale $(0,53)$.

Em Remígio os maiores valores de IR, VU e RFC foram atribuídos as mesmas espécies, $S$. obtusifolium (IR $=2 ; \mathrm{VU}=0,64 ; \mathrm{RFC}=0,24)$, C. pyramidale $(1,5 ; 0,90 ; 0,36)$, A. cearensis $(1,35 ; 0,62 ; 0,33)$ e $M$. urundeuva $(1,28 ; 0,79 ; 0,55)$, havendo apenas alterações na colocação das espécies na lista de cada método, por exemplo, S. obtusifolium obteve a maior pontuação na IR, a terceira maior no VU e a quarta maior na FRC.

Tabela 3. Principais espécies de plantas medicinais ranqueadas pelos métodos de Importância Relativa (IR), Valor de Uso (VU) e Frequência Relativa de Citação (FRC) nas comunidades estudadas nos Municípios de Solânea e Remígio, Estado da Paraíba, Nordeste do Brasil.

\begin{tabular}{|l|c|c|c|c|c|c|}
\hline \multicolumn{1}{|c|}{ Espécie } & \multicolumn{3}{c|}{ Solânea } & \multicolumn{3}{c|}{ Remígio } \\
\hline \multicolumn{1}{|c|}{} & IR & VU & FRC & IR & VU & FRC \\
\hline Myracrodruon urundeuva & $1^{\circ}$ & $1^{\circ}$ & $1^{\circ}$ & $4^{\circ}$ & $2^{\circ}$ & $1^{\circ}$ \\
\hline Monteverdia rigida & $2^{\circ}$ & - & - & - & - & - \\
\hline Sideroxylon obtusifolium & $3^{\circ}$ & $4^{\circ}$ & $5^{\circ}$ & $1^{\circ}$ & $3^{\circ}$ & $4^{\circ}$ \\
\hline Anadenanthera colubrina & $4^{\circ}$ & - & - & - & - & - \\
\hline Cenostigma pyramidale & $4^{\circ}$ & $3^{\circ}$ & $3^{\circ}$ & $2^{\circ}$ & $1^{\circ}$ & $2^{\circ}$ \\
\hline Ximenia americana & $4^{\circ}$ & - & - & - & - & - \\
\hline Commiphora leptophloeos & $5^{\circ}$ & - & - & - & - & - \\
\hline Amburana cearensis & - & $2^{\circ}$ & $2^{\circ}$ & $3^{\circ}$ & $4^{\circ}$ & $3^{\circ}$ \\
\hline Schinopsis brasiliensis & - & - & - & - & - & - \\
\hline Handroanthus impetiginosa & - & - & - & - & - & - \\
\hline Croton blanchetianus & - & $5^{\circ}$ & $4^{\circ}$ & - & - & - \\
\hline Bauhinia cheilantha & - & - & - & $5^{\circ}$ & - & - \\
\hline Hymenaea courbaril & - & - & - & $5^{\circ}$ & $5^{\circ}$ & $4^{\circ}$ \\
\hline Ziziphus joazeiro & - & - & - & - & - & $5^{\circ}$ \\
\hline
\end{tabular}

Mesorregião da Borborema (Soledade, Congo e Cabaceiras). Nas Tabelas 4 e 5 é encontrado o comparativo das espécies de maior destaque conforme cada índice nos Municípios da Borborema. Na Comunidade Barrocas, em Soledade, os maiores valores de IR, VU e FRC foram para Ximenia americana $\mathrm{L}$. (ameixa, IR = 1,83; VU = 0,86; FRC = 0,67), Cnidoscolus quercifolius Pohl. (favela, 1,74; 0,57, 0,53), Bauhinia cheilantha (Bong.) Steud. (mororó, 1,73; 0,57; 0,47), S. obtusifolium $(1,65 ; 0,71 ; 0,73)$ e M. urundeuva $(1,56 ; 0,48$; $0,47)$.

Na Comunidade Cachoeira, também em Soledade, o destaque foi para M. rigida (IR $=2 ; \mathrm{VU}=0,96 ; \mathrm{FRC}=0,48), B$. cheilantha $(1,94 ; 1,08 ; 0,59)$, A. cearensis $(1,71 ; 1,17 ; 0,59)$, 
S. obtusifolium $(1,57 ; 1,04 ; 0,66)$, C. quercifolius $(1,57 ; 1,42 ; 0,79)$, M. urundeuva $(1,51$; $0,67 ; 0,48)$ e $X$. americana $(1,51 ; 1,42 ; 0,72)$.

No Congo, sobressaíram S. obtusifolium ( $\mathrm{IR}=2 ; \mathrm{VU}=1,41 ; \mathrm{FRC}=0,76$ ), $X$. americana $(1,50 ; 0,64 ; 0,32), M$. urundeuva $(1,49 ; 0,65 ; 0,43), C$. quercifolius $(1,17 ; 0,29$; $0,15)$ e Ziziphus joazeiro Mart. (juazeiro; 1,$10 ; 0,59 ; 0,38$ ). É observado que $C$. quercifolius, embora tenha um valor alto de IR obteve valores baixos de UV e FRC. Em relação ainda ao VU e FRC duas espécies obtiveram certo destaque, embora não o tenham tido na IR, C. pyramidale (VU $=0,62$; FRC $=0,42$ ) e Mimosa tenuiflora (Willd.) Poir. (jurema preta; $0,47 ; 0,34)$.

Em Cabaceiras sobressaíram S. obtusifolium (IR = 2; VU = 0,96; FRC = 0,74), M. urundeuva (1,87; 0,77; 0,59); Pseudobombax marginatum (A. St.-Hil., Juss. \& Cambress.) A. Robyns (imbiratã; 1,$80 ; 0,33 ; 0,25)$, B. cheilantha $(1,53 ; 0,11 ; 0,08)$ e Commiphora leptophloeos (Mart.) J.B. Gillett (umburana; 1,50; 0,37; 0,33). P. marginatum e B. cheilantha apesar do destaque na IR obtiveram valores mais baixos no VU e FRC. Por outro lado, espécies que não tiveram tanto destaque no IR se sobressaíram no VU e/ou FRC, sendo C. pyramidale $(0,59 ; 0,45)$, Jatropha mollissima (Pohl) Baill. (pinhão brabo) (VU = 0,50; FRC $=0,31)$ e $Z$. joazeiro $(0,47 ; 0,35)$.

Tabela 4. Principais espécies de plantas medicinais ranqueadas pelos métodos de Importância Relativa (IR), Valor de Uso (VU) e Frequência Relativa de Citação (FRC) nas comunidades estudadas, nos Municípios de Cabaceiras e Congo, Estado da Paraíba, nordeste do Brasil.

\begin{tabular}{|l|c|c|c|c|c|c|}
\hline & \multicolumn{3}{|c|}{ Cabaceiras } & \multicolumn{3}{c|}{ Congo } \\
\hline Espécie & IR & VU & FRC & IR & VU & FRC \\
\hline Sideroxylon obtusifolium & $1^{\circ}$ & $1^{\circ}$ & $1^{\circ}$ & $1^{\circ}$ & $1^{\circ}$ & $1^{\circ}$ \\
\hline Myracrodruon urundeuva & $2^{\circ}$ & $2^{\circ}$ & $2^{\circ}$ & $3^{\circ}$ & $2^{\circ}$ & $2^{\circ}$ \\
\hline Pseudobombax marginatum & $3^{\circ}$ & - & - & - & - & - \\
\hline Bauhinia cheilantha & $4^{\circ}$ & - & - & - & - & - \\
\hline Commiphora leptophloeos & $5^{\circ}$ & - & $5^{\circ}$ & - & - & - \\
\hline Cenostigma pyramidale & - & $3^{\circ}$ & $3^{\circ}$ & - & $4^{\circ}$ & $3^{\circ}$ \\
\hline Jatropha mollissima & - & $4^{\circ}$ & - & - & - & - \\
\hline Ziziphus joazeiro & - & $5^{\circ}$ & $4^{\circ}$ & $5^{\circ}$ & $5^{\circ}$ & $4^{\circ}$ \\
\hline Ximenia americana & - & - & - & $2^{\circ}$ & $3^{\circ}$ & - \\
\hline Cnidoscolus quercifolius & - & - & - & $4^{\circ}$ & - & - \\
\hline Mimosa tenuiflora & - & - & - & - & - & $5^{\circ}$ \\
\hline
\end{tabular}

Tabela 5. Principais espécies de plantas medicinais ranqueadas pelos métodos Importância Relativa (IR), Valor de Uso (VU) e Frequência Relativa de Citação (FRC) nas comunidades estudadas, nos Municípios no Município de Soledade, Estado da Paraíba, nordeste do Brasil.

\begin{tabular}{|l|c|c|c|c|c|c|}
\hline & \multicolumn{4}{|c|}{ Soledade - Barrocas } & \multicolumn{3}{c|}{ Soledade - Cachoeira } \\
\hline Espécie & IR & VU & FRC & IR & VU & FRC \\
\hline Ximenia americana & $1^{\circ}$ & $1^{\circ}$ & $2^{\circ}$ & $5^{\circ}$ & $1^{\circ}$ & $2^{\circ}$ \\
\hline Cnidoscolus quercifolius & $2^{\circ}$ & $4^{\circ}$ & $3^{\circ}$ & $4^{\circ}$ & $1^{\circ}$ & $1^{\circ}$ \\
\hline Bauhinia cheilantha & $3^{\circ}$ & $4^{\circ}$ & $4^{\circ}$ & $2^{\circ}$ & $3^{\circ}$ & $4^{\circ}$ \\
\hline Sideroxylon obtusifolium & $4^{\circ}$ & $2^{\circ}$ & $1^{\circ}$ & $4^{\circ}$ & $4^{\circ}$ & $3^{\circ}$ \\
\hline Myracrodruon urundeuva & $5^{\circ}$ & - & $4^{\circ}$ & $5^{\circ}$ & - & $5^{\circ}$ \\
\hline Amburana cearensis & - & $3^{\circ}$ & $4^{\circ}$ & $3^{\circ}$ & $2^{\circ}$ & $4^{\circ}$ \\
\hline Monteverdia rigida & - & $4^{\circ}$ & $5^{\circ}$ & $1^{\circ}$ & $5^{\circ}$ & $5^{\circ}$ \\
\hline Cenostigma pyramidale & - & $5^{\circ}$ & $4^{\circ}$ & - & - & - \\
\hline Jatropha mollissima & - & - & $5^{\circ}$ & - & - & - \\
\hline Ziziphus joazeiro & - & - & $5^{\circ}$ & - & - & - \\
\hline
\end{tabular}

Rev. Bras. Gest. Amb. Sustent., 2021, vol. 9, n. 21, p. 37-57. 
Mesorregião do Sertão (São Mamede, Lagoa e Itaporanga). A Tabela 6 compara as espécies de maior relevância nos municípios do Sertão. Em São Mamede as espécies que se sobressaíram foram $Z$. joazeiro ( $\mathrm{IR}=1,85 ; \mathrm{VU}=0,94 ; \mathrm{FRC}=0,51)$, $S$. obtusifolium $(1,85$; 0,50; 0,22), Libidibia ferrea (Mart. ex Tul.) L.P. Queiroz (jucá) (1,85; 0,78; 0,22), C. quercifolius $(1,83 ; 1,33 ; 0,70)$ e M. urundeuva $(1,75 ; 0,67 ; 0,27)$. Embora tenham se destacado na IR e no VU, os valores de FRC para S. obtusifolium, L. férrea e M. urundeuva foram relativamente baixos, por outro lado, algumas espécies que com IR e VU mais baixos obtiveram resultados mais altos no FRC, sendo M. tenuiflora $(\mathrm{FRC}=0,35)$, J. mollissima $(0,32)$ e Croton blanchetianus Baill. (marmeleiro; 0,30).

Em Lagoa o destaque foi para $S$. obtusifolium ( $\mathrm{IR}=2 ; \mathrm{VU}=1,09 ; \mathrm{FRC}=0,59$ ), M. urundeuva $(1,70 ; 0,77 ; 0,47)$, Indet. 1 (jaramataia; 1,48; 0,27; 0,12); M. tenuiflora $(1,44$; $0,86 ; 0,56)$ e $X$. americana $(1,33 ; 0,35 ; 0,23)$. Indet. 1 e X. americana, apesar da relevância no valor da IR obtiveram valores menores de VU e FRC, no entanto, outras espécies que obtiveram resultados mais baixos de IR obtiveram melhores resultados de VU e FRC: C. blanchetianus (VU = 0,73; FRC = 0,53), Combretum fruticosum (Loefl) Stuntz (mufumbo; $\mathrm{VU}=0,68 ; \mathrm{FRC}=0,42)$ e $A$. cearensis $(0,67 ; 0,47)$.

Em Itaporanga as espécies mais versáteis foram M. urundeuva $(\mathrm{IR}=2 ; \mathrm{VU}=0,47)$, Jocasanda momosifolia (Mart ex DC) Standl. (caroba; 1,34; 0,20; 0,13), C. leptophloeos $(1,34 ; 0,27 ; 0,27)$, C. fruticosum $(1,34 ; 0,33 ; 0,33)$, C. pyramidale $(1,34 ; 0,73 ; 0,47)$ e Tocoyena formosa (Cham. \& Schltdle) K. Schum (jenipapo brabo; 1,34; 0,27; 0,13). Entre estas espécies, apenas $T$. formosa obteve maior relevância na IR porém valores baixos de VU e FRC. $X$. americana obteve baixa IR $(0,66)$, no entanto obteve valores mais altos para VU e FRC (ambos 0,40).

Tabela 6. Principais espécies de plantas medicinais ranqueadas pelos métodos de Importância Relativa (IR), Valor de Uso (VU) e Frequência Relativa de Citação (FRC) nas comunidades estudadas, nos Municípios de São Mamede, Lagoa e Itaporanga, Estado da Paraíba, Nordeste do Brasil.

\begin{tabular}{|l|c|c|c|c|c|c|c|c|c|}
\hline & \multicolumn{3}{|c|}{ São Mamede } & \multicolumn{3}{c|}{ Lagoa } & \multicolumn{3}{c|}{ Itaporanga } \\
\hline Espécie & IR & VU & FRC & IR & VU & FRC & IR & VU & FRC \\
\hline Libidibia ferrea & $1^{\circ}$ & $3^{\circ}$ & - & - & - & - & - & - & - \\
\hline Ziziphus joazeiro & $1^{\circ}$ & $2^{\circ}$ & $2^{\circ}$ & - & - & $5^{\circ}$ & - & - & - \\
\hline Sideroxylon obtusifolium & $1^{\circ}$ & $5^{\circ}$ & - & $1^{\circ}$ & $1^{\circ}$ & $1^{\circ}$ & - & - & - \\
\hline Cnidoscolus quercifolius & $2^{\circ}$ & $1^{\circ}$ & $1^{\circ}$ & - & - & - & - & - & - \\
\hline Myracrodruon urundeuva & $3^{\circ}$ & $4^{\circ}$ & - & $2^{\circ}$ & $3^{\circ}$ & $4^{\circ}$ & $1^{\circ}$ & $2^{\circ}$ & $3^{\circ}$ \\
\hline Mimosa tenuiflora & $4^{\circ}$ & $4^{\circ}$ & $3^{\circ}$ & $4^{\circ}$ & $2^{\circ}$ & $2^{\circ}$ & - & - & - \\
\hline Combretum fruticosum & $5^{\circ}$ & - & - & - & $5^{\circ}$ & - & $2^{\circ}$ & $4^{\circ}$ & $3^{\circ}$ \\
\hline Jatropha mollissima & - & - & $4^{\circ}$ & - & - & - & - & - & - \\
\hline Croton blanchetianus & - & - & $5^{\circ}$ & - & $4^{\circ}$ & $3^{\circ}$ & - & - & - \\
\hline Indet. 1 & - & - & - & $3^{\circ}$ & - & - & - & - & - \\
\hline Ximenia americana & - & - & - & $5^{\circ}$ & - & - & - & $3^{\circ}$ & $2^{\circ}$ \\
\hline Amburana cearensis & - & - & - & - & - & $4^{\circ}$ & - & - & - \\
\hline Jocasanda momosifolia & - & - & - & - & - & - & $2^{\circ}$ & - & $5^{\circ}$ \\
\hline Commiphora leptophloeos & - & - & - & - & - & - & $2^{\circ}$ & $5^{\circ}$ & $4^{\circ}$ \\
\hline Cenostigma pyramidale & - & - & - & - & - & - & $2^{\circ}$ & $1^{\circ}$ & $1^{\circ}$ \\
\hline Tocoyena formosa & - & - & - & - & - & - & $2^{\circ}$ & - & $5^{\circ}$ \\
\hline
\end{tabular}

\section{Discussão}

O número de espécies utilizadas nas comunidades estudadas, no presente estudo, no semiárido da Paraíba, é relativamente menor do que o observado em outros estudos 
realizados em outras áreas secas (Cartaxo et al., 2010; Lozano et al., 2014; Abbas et al., 2017), e esta distinção pode se dá em função de diversos fatores específicos da nossa pesquisa, como por exemplo as variações culturais e ambientais bem como os critérios adotados, visto que, adotamos e registramos apenas informações de espécies lenhosas e nativas da Caatinga.

Fabaceae e Euphorbiaceae são famílias que costumam ser bastante representativas em estudos etnobotânicos na Caatinga (Macedo et al., 2018; Caetano et al., 2020; Campos e Albuquerque, 2021). A relevância dessas famílias, possivelmente, se deve à sua diversidade de espécies e ampla distribuição em áreas de Caatinga, como têm sido observado em inventários fitossociológicos realizados no semiárido paraibano (Pereira et al., 2002; Trovão et al., 2010).

Embora os índices de Importância Relativa, Valor de Uso e Frequência Relativa de Citação sejam formulados com finalidades distintas, foi possível observar uma correspondência entre estes índices nas áreas estudadas, com pequenas exceções, de espécies que obtiveram valores altos em um e baixos em outro. Outros estudos de comparação de métodos quantitativos também têm observado uma correspondência na listagem de espécies de destaque em cada método, como Valor de Uso e Importância Relativa (Albuquerque et al., 2006), e Valor de Uso e listagem de inventário in situ(Lucena et al., 2013). Em outros casos de comparação de métodos, distinções maiores entre as listagens foram observadas, como na comparação do Valor de Uso com o Índice de Prioridade de Conservação (Lucena et al., 2013), e em comparações de Importância Relativa com Valor de Uso e Frequência Relativa de Citações (Vitalini et al., 2013).

Também no sentido de comparar métodos quantitativos, muitos outros estudos etnobotânicos vêm sendo feitos. Em Bangladesh, por exemplo, não foi encontrada uma correspondência entre as principais espécies no índice de Importância Relativa em comparação à Frequência Relativa de Citação (Faruque et al., 2018). Em um estudo no Paquistão também não foi observada correspondência entre as principais espécies na lista do Valor de Uso e na Importância Relativa (Yaseen et al., 2015), a partir de outro estudo, também no Paquistão, foi possível notar uma correspondência entre as principais espécies na Frequência Relativa de Citação, Valor de Uso e Importância Relativa (Amjad et al., 2017). Também foi possível notar uma correspondência das principais espécies alimentícias utilizadas na Indonésia, quando avaliadas sob diferentes índices quantitativos (Sujarwo e Caneva, 2016). Todos esses estudos evidenciam a possibilidade e importância da triangulação de resultados alcançados por diferentes métodos com o objetivo de compreender melhor a relação das populações humanas com os recursos naturais, assim como foi também realizado em nosso estudo.

M. urundeuvae S. obtusifoliumse apresentaram como espécies de destaque em todos os índices, e em quase todas as áreas estudadas, com exceção apenas de Itaporanga, onde não foram registrados usos para $S$. obtusifolium, o que se deve a inexistência desta espécie na vegetação local. Tais dados condizem com outros estudos, onde ambas as espécies apresentam alta relevância para as pessoas das comunidades estudadas (Almeida e Albuquerque, 2002; Ferraz et al., 2005).

M. urundeuvaé uma espécie amplamente utilizada na medicina tradicional no Nordeste do Brasil (Santos et al., 2018; Silva et al., 2019; Ferreira, 2021b), bem como para outros usos diversos como combustível, construção, forragem, tecnologia e veterinário (Ramos et al., 2008; Sousa et al., 2012; Barros et al., 2016; Alves et al., 2019; Barbosa et al., 2020). De modo semelhante, S. obtusifolium também apresenta usos madeireiros, além do medicinal (Pedrosa et al., 2012). 0 destaque destas espécies nas distintas áreas e nos diferentes métodos quantitativos pode ser um indício da uma possível pressão de uso sobre estas espécies, ainda mais considerando os registros de usos madeireiros delas, que também pode ser um fator de influência sobre uma maior pressão de uso local. 


\section{Conclusões}

A partir dos dados obtidos, no presente estudo, foi possível observar uma correspondência entre as espécies de destaque nos índices de Importância Relativa, Valor de Uso, e Frequência Relativa de Citação nas diferentes comunidades estudadas, o que pode representar uma difusão do conhecimento sobre plantas medicinais entre as áreas estudadas.

Nossos resultados também evidenciam a importância da triangulação de métodos qualitativos e quantitativos para um olhar mais robusto sobre a relação das populações humanas com os recursos naturais, especialmente contribuindo em ações conservacionistas e políticas públicas locais e regionais.

M. urudeuvae e S. obtusifolium apresentaram grande relevância em todas as áreas estudadas, com exceção apenas de Itaporanga, onde não foi registrado o uso de S. obtusifolium. Estes dados apontam para a necessidade de estabelecer estratégias de uso sustentável destas espécies, de modo a evitar uma pressão excessiva sobre suas populações. Além disso, também acreditamos ser importante a realização de mais estudos com foco em ações conservacionistas, além de poder fornecer informações para ações de políticas públicas locais e regionais.

\section{Conflito de interesses}

Os autores declaram não haver conflito de interesses.

\section{Referências}

Abbas, Z.; Khan, S. M.; Alam, J.; Khan, S. W.; Abbasi, A. M. Medicinal plants used by inhabitants of the Shigar Valley, Baltistan Region of Karakorum Range-Pakistan. Journal

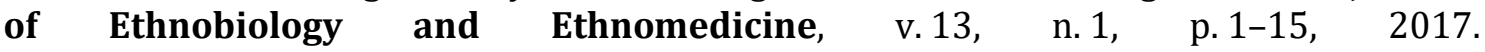
https://doi.org/10.1186/S13002-017-0172-9

Albuquerque, U. P.; Lucena, R. F. P.; Alencar, N. L. Métodos e técnicas para coleta de dados etnobiológicos. In: Albuquerque, U. P.; Lucena, R. F. P.; Cunha, L. V. F. C. (Eds.). Métodos e técnicas na pesquisa etnobiológica e etnoecológica. Recife: NUPEEA, 2010. p. 39-64.

Albuquerque, U. P.; Lucena, R. F. P.; Monteiro, J. M.; Florentino, A. T. N.; Almeida, C. F. C. B. R. Evaluating two quantitative ethnobotanical techniques. Ethnobotany Reserach \& Applications, v. 4, p. 51-60, 2006.

Alencar, N. L.; Araújo, T. A. S.; Amorim, E. L. C.; Albuquerque, U. P. The inclusion and selection of medicinal plants in traditional pharmacopoeias-evidence in support of the diversification hypothesis. Economic Botany, v.64, n. 1, p.68-79, 2010. https://doi.org/10.1007/s12231-009-9104-5

Almeida, C. F. C. B. R.; Albuquerque, U. P. Uso e conservação de plantas e animais medicinais no Estado de Pernambuco (Nordeste do Brasil): um estudo de caso. Interciencia, v. 27, n. 6, p. 276-285, 2002.

Alves, C. A. B.; Ribeiro, J. E. S.; Guerra, N. M.; Nunes, M. M.; Barbosa, E. U. G.; Nunes, E. N.; Carvalho, T. K. N.; Lucena, C. M.; Souto, J. S.; Lucena, R. F. P. Conhecimento botânico local sobre Myracrodruon urundeuva Allemão em seis comunidades rurais do Semiárido da Paraíba, Nordeste do Brasil. Gaia Scientia, v. 13, n. 1, p. 60-79, 2019.

Amjad, M. S.; Qaeem, M. F.; Ahmad, I.; Khan, S. U.; Chaudhari, S. K.; Zahid Malik, N.; Shaheen, H.; Khan, A. M. Descriptive study of plant resources in the context of the ethnomedicinal relevance of indigenous flora: A case study from Toli Peer National Park, Azad Jammu and 
Kashmir, Pakistan. PLoS ONE, v. 12, n. 2, e0171896, 2017. https://doi.org/10.1371/ journal.pone.0171896

Barbosa, E. U. G.; Carvalho, T. K. N.; Ferreira, E. C.; Santos, S. S.; Lucena, R. F. P. Conhecimento botânico local de agricultores do Semiárido do Estado da Paraíba, Nordeste do Brasil. Polibotánica, n. 50, p. 191-208, 2020. https://doi.org/10.18387/ polibotanica.50.13

Barros, F. N.; Nascimento, V. T.; Medeiros, P. M. Ethnobotany and population status of Myracrodruon urundeuva Allemão in rural Northeastern Brazil. Economic Botany, v. 70, n. 1, p. 79-84, 2016. https://doi.org/10.1007/s12231-015-9329-4

Batista, W. F. M.; Santos, K. P. P.; Barros, R. F. M. Conhecimento tradicional numa comunidade rural do Nordeste brasileiro. Gaia Scientia, v. 11, n. 1, p. 225-252, 2017.

Bennett, B. C.; Prance, G. T. Introduced plants in the indigenous pharmacopoeia of Northern South America. Economic Botany, v. 54, n. 1, p. 90-102, 2000.

Bermúdez, A.; Oliveira-Miranda, M. A.; Velázquez, D. La investigación etnobotánica sobre plantas medicinales: una revisión de sus objetivos y enfoques actuales. Interciencia, v. 30, n. 8, p. 453-459, 2005.

Bhat, J. A.; Kumar, M.; Bussmann, R. W. Ecological status and traditional knowledge of medicinal plants in Kedarnath Wildlife Sanctuary of Garhwal Himalaya, India. Journal of Ethnobiology and Ethnomedicine, v. 9, n. 1, 2013. https://doi.org/10.1186/1746-42699-1

Bussmann, R. W.; Paniagua Zambrana, N. Y.; Sikharulidze, S.; Kikvidze, Z.; Kikodze, D.; Tchelidze, D.; Khutsishvili, M.; Batsatsashvili, K.; Hart, R. E. A comparative ethnobotany of Khevsureti, Samtskhe-Javakheti, Tusheti, Svaneti, and Racha-Lechkhumi, Republic of Georgia (Sakartvelo), Caucasus. Journal of Ethnobiology and Ethnomedicine, v. 12, n. 1, 2016. https://doi.org/10.1186/s13002-016-0110-2

Caetano, R. A.; Albuquerque, U. P.; Medeiros, P. M. What are the drivers of popularity and versatility of medicinal plants in local medical systems? Acta Botanica Brasilica, v. 34, n. 2, p. 256-265, 2020. https://doi.org/10.1590/0102-33062019abb0233

Campos, J. L. A.; Albuquerque, U. P. Indicators of conservation priorities for medicinal plants from seasonal dry forests of Northeastern Brazil. Ecological Indicators, v. 121, p. 1470-160, 2021. https://doi.org/10.1016/j.ecolind.2020.106993

Cartaxo, S. L.; Almeida Souza, M. M.; Albuquerque, U.P. Medicinal plants with bioprospecting potential used in semi-arid Northeastern Brazil. Journal of Ethnopharmacology, $\quad$ v. 131, $\quad$ n. 2, p. 326-342, 2010. https://doi.org/10.1016/ j.jep.2010.07.003

Carvalho, T. K. N.; Sousa, R. F. P.; Meneses, S. S. S.; Ribeiro, J. P. O.; Felix, L. P.; Lucena, R. F. P. Plantas usadas por uma comunidade rural na Depressão Sertaneja no Nordeste do Brasil. Revista de Biologia e Farmácia, v. esp., p. 92-120, 2012.

Coutinho, P. C.; Soares, Z. A.; Ferreira, E. C.; Souza, D. V.; Oliveira, R. S.; Lucena, R. F. P. Knowledge and use of medicinal plants in the Semiarid Region of Brazil. Brazilian Journal of Biological Sciences, v. 2, n. 3, p. 51-74, 2015. https://doi.org/10.21472/bjbs.020306

Dantas, I. C.; Guimarães, F. R. Plantas medicinais comercializadas no Município de Campina Grande, PB. Revista de Biologia e Farmácia, v. 1, n. 1, 2007. 
Dhar, U.; Rawal, R. S.; Upreti, J. Setting priorities for conservation of medicinal plants: A case study in the Indian Himalaya. Biological Conservation, v. 95, p. 57-65, 2000. https://doi.org/10.1016/S0006-3207(00)00010-0

Faruque, M. O.; Uddin, S. B.; Barlow, J. W.; Hu, S.; Dong, S.; Cai, Q.; Li, X.; Hu, X. Quantitative ethnobotany of medicinal plants used by indigenous communities in the Bandarban District of Bangladesh. Frontiers in Pharmacology, v. 9, 2018. https://doi.org/10.3389/fphar.2018.00040

Ferraz, J. S. F.; Albuquerque, U. P.; Meunier, I. M. J. Valor do uso e estrutura da vegetação lenhosa às margens do Riacho do Navio, Floresta, PE, Brasil. Acta Botanica Brasilica, v. 20, p. 125-134. 2006. https://doi.org/10.1590/S0102-33062006000100012

Ferreira, E. C.; Anselmo, M. G. V.; Guerra, N. M.; Lucena, C. M.; Felix, C. M. P.; Bussmann, R. W.; Paniagua-Zambrana, N. Y.; Lucena, R. F. P. Local knowledge and use of medicinal plants in a rural community in the Agreste of Paraíba, Northeast Brazil. Evidence-Based Complementary and Alternative Medicine, v. 2021, 2021. https://doi.org/10.1155/ 2021/9944357

Ferreira, E. C.; Lucena, R. F. P.; Bussmann, R. W.; Paniagua-Zambrana, N. Y.; Cruz, D. D. Temporal assessment of the medicinal plants trade in public markets of the State of Paraíba, Northeastern Brazil. Journal of Ethnobiology and Ethnomedicine, v. 17, n. 70, 2021. https://doi.org/10.1186/S13002-021-00496-3

Gera, M.; Blsht, N. S.; Rana, A. K. Market information system for sustainable management of medicinal plants. Indian Forester, v. 129, n. 1, p. 102-108, 2003.

Guerra, N. M.; Carvalho, T. K. N.; Ribeiro, J. E. S.; Ribeiro, J. P. O.; Barbosa, A. R.; Limam, J. R. F.; Alves, C. A. B.; Oliveira, R. S.; Lucena, R. F. P. Ecological apparency hypothesis and plant utility in the Semiarid Region of Brazil. Ethnobotany Research and Applications, v. 14, p. 423-435, 2015.

Guerra, N. M.; Ribeiro, J. P. S.; Carvalho, T. K. N.; Pedrosa, K. M.; Félix, L. P.; Lucena, R. F. P. Usos locais de espécies vegetais nativas em uma comunidade rural no semiárido nordestino (São Mamede, Paraíba, Brasil). Revista de Biologia e Farmácia, v. esp., p. 184-210, 2012.

IBGE - Instituto Brasileiro de Geografia e Estatística. Censo 2010. Disponível em: <http://www.ibge.gov.br>. Acesso em: 15 maio 2021.

ICD - International Classification of Deasese. ICD-10 Version: 2010. Disponível em: <http://apps.who.int/classifications/ icd10/browse/2010/en>. Acesso em: 23 jun. 2021.

Jha, A. K. Medicinal plants: Poor regulation blocks conservation. Economic and Political Weekly, v. 30, n. 51, p. 3270-3270, 1995.

Leite, A. P.; Pedrosa, K. M.; Lucena, C. M.; Carvalho, T. K. N.; Félix, L. P.; Lucena, R. F. P. Uso e conhecimento de espécies em uma comunidade rural no Vale do Piancó (Paraíba, Nordeste, Brasil). Revista de Biologia e Farmácia, v. esp., p. 133-157, 2012.

Lozano, A.; Araújo, E. L.; Medeiros, M. F. T.; Albuquerque, U. P. The apparency hypothesis applied to a local pharmacopoeia in the Brazilian Northeast. Journal of Ethnobiology and Ethnomedicine, v. 10, n. 2, 2014. https://doi.org/10.1186/1746-4269-10-2

Lucena, R. F. P.; Lucena, C. M.; Araújo, E. L.; Alves, A. G. C.; Albuquerque, U. P. Conservation priorities of useful plants from different techniques of collection and analysis of ethnobotanical data. Anais da Academia Brasileira de Ciências, v. 85, n. 1, p. 169-186, 2013. https://doi.org/10.1590/S0001-37652013005000013 
Lucena, R. F. P.; Medeiros, P. M.; Araújo, E. L.; Alves, A. G. C.; Albuquerque, U. P. The ecological apparency hypothesis and the importance of useful plants: An assessment based on value-use. Journal of Environmental Management, v. 96, p. 106-115, 2012.

Lucena, R. F. P.; Pedrosa, K. M.; Carvalho, T. K. N.; Guerra, N. M.; Ribeiro, J. E. S.; Ferreira, E. C. Conhecimento local e uso de espécies vegetais nativas da região da Serra de Santa Catarina, Paraíba, Nordeste do Brasil. FLOVET, v. 1, n. 9, p. 158-179, 2017.

Macedo, J. G. F.; Menezes, I. R. A.; Ribeiro, D. A.; Santos, M. D. O.; Mâcedo, D. G.; Macêdo, M. J. F.; Almeida, B. V.; Oliveira, L. G. S; Leite, C. P.; Souza, M. M. A. Analysis of the variability of therapeutic indications of medicinal species in the Northeast of Brazil: Comparative study. Evidence-Based Complementary and Alternative Medicine, v. 2018, 2018. https://doi.org/10.1155/2018/6769193

Nunes, A. T.; Lucena, R. F. P.; Santos, M. V. F.; Albuquerque, U. P. Local knowledge about fodder plants in the Semi-Arid Region of Northeastern Brazil. Journal of Ethnobiology and Ethnomedicine, v. 11, n. 12, 2015. https://doi.org/10.1186/1746-4269-11-12

Nunes, E. N.; Guerra, N. M.; Arévalo-Marín, E.; Alves, C. A. B.; Nascimento, V. T.; Cruz, D. D.; Ladio, A. H.;Silva, S. M. S; Oliveira, R. S.; Lucena, R. F. P. Local botanical knowledge of native food plants in the semiarid region of Brazil. Journal of Ethnobiology and Ethnomedicine, v. 14, n. 49, 2018. https://doi.org/10.1186/s13002-018-0249-0

Oliveira, F. C.; Albuquerque, U. P.; Fonseca-Kruel, V. S.; Hanazaki, N. Avanços nas pesquisas etnobotânicas no Brasil. Acta Botanica Brasilica, v. 23, n. 2, p. 590-605, 2009. https://doi.org/10.1590/S0102-33062009000200031

Pedrosa, K. M.; Gomes, D. S.; Lucena, C. M.; Pereira, D. D.; Silviano, G. S.; Lucena, R. F. P. Uso e disponibilidade local de Sideroxylon obtusifolium (Roem. \& Schult.) T.D. Penn. (quixabeira) em três regiões da Depressão Sertaneja da Paraíba, Nordeste do Brasil. Revista de Biologia e Farmácia, v. esp., p. 158-183, 2012.

Pereira, I. M.; Andrade, L. A.; Barbosa, M. R. V.; Sampaio, E. V. S. B. Composição florística e análise fitossociológica do componente arbustivo-arbóreo de um remanescente florestal no Agreste Paraibano. Acta Botanica Brasilica, v. 16, n. 3, p. 357-369, 2002.

Phillips, O.; Gentry, A. H. The useful plants of Tambopata, Peru: I. Statistical hypotheses tests with a new quantitative technique. Economic Botany, v. 47, n. 1, p. 15-32, 1993a. https://doi.org/10.1007/BF02862203

Phillips, O.; Gentry, A. H. The useful plants of Tambopata, Peru: II. Additional hypothesis testing in quantitative ethnobotany. Economic Botany, v. 47, n. 1, p. 33-43, 1993b. https://doi.org/10.1007/BF02862204

Ramos, M. A.; Medeiros, P. M.; Almeida, A. L. S.; Feliciano, A. L. P.; Albuquerque, U. P. Use and knowledge of fuel wood in an area of Caatinga vegetation in NE Brazil. Biomass and Bioenergy, v. 32, n. 6, p. 510-517, 2008. https://doi.org/10.1016/j.biombioe.2007.11.015

Ribeiro, D. A.; Macedo, D. G.; Oliveira, L. G. S.; Santos, M. O.; Almeida, B. V.; Macedo, J. G. F.; Macêdo, M. J. F.; Souza, R. K. D.; Araújo, T. M. S.; Souza, M. M. A. Conservation priorities for medicinal woody species in a cerrado area in the Chapada do Araripe, Northeastern Brazil. Environment, Development and Sustainability, v. 21, n. 1, p.61-77, 2019. https://doi.org/10.1007/s10668-017-0023-9

Ribeiro, J. E. S.; Carvalho, T. K. N.; Ribeiro, J. P. O.; Guerra, N. M.; Silva, N.; Pedrosa, K. M.; Alves, C. A. B.; Sousa Júnior, S. P.; Souto, J. S.; Nunes, A. T.; Lima, J. R. F.; Oliveira, R. S.; Lucena, R. F. P. Ecological apparency hypothesis and availability of useful plants: Testing different use values. Ethnobotany Research and Applications, v. 12, p. 415-432, 2014. 
Ribeiro, R. V.; Bieski, I. G. C.; Balogun, S. O.; Martins, D. T. O. Ethnobotanical study of medicinal plants used by ribeirinhos in the North Araguaia Microregion, Mato Grosso,

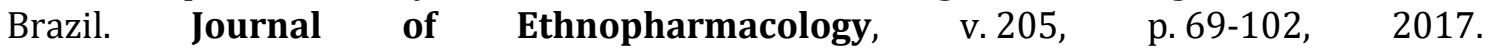
https://doi.org/10.1016/j.jep.2017.04.023

Ritter, M. R.; Silva, T. C.; Araújo, E. L.; Albuquerque, U. P. Bibliometric analysis of ethnobotanical research in Brazil (1988-2013). Acta Botanica Brasilica, v. 29, n. 1, p. 113-119, 2015. https://doi.org/10.1590/0102-33062014abb3524

Rossato, S. C.; Leitão-Filho, H. F.; Begossi, A. Ethnobotany of caiçaras of the Atlantic Forest Coast (Brazil). Economic Botany, v. 53, n. 4, p. 387-395, 1999. https://doi.org/10.1007/ BF02866716

Santos, M. O.; Ribeiro, D. A.; Macêdo, D. G.; Macêdo, M. J. F.; Macedo, J. G. F.; Lacerda, M. N. S.; Macêdo, M. S.; Souza, M. M. A. Medicinal plants: Versatility and concordance of use in the Caatinga area, Northeastern Brazil. Anais da Academia Brasileira de Ciências, v. 9, n. 3, 2018. https://doi.org/10.1590/0001-3765201820170594

Silva, N. F.; Hanazaki, N.; Albuquerque, U. P.; Campos, J. L. A.; Feitosa, I. S.; Lima, E. A. Local knowledge and conservation priorities of medicinal plants near a protected area in Brazil. Evidence-Based Complementary and Alternative Medicine, v. 2019, 2019. https://doi.org/10.1155/2019/8275084

Silva, N.; Lucena, R. F. P.; Lima, J. R. F.; Lima, G. D. S.; Carvalho, T. K. N.; Sousa Júnior, S. P.; Alves, C. A. B. Conhecimento e uso da vegetação nativa da Caatinga em uma comunidade rural da Paraíba, Nordeste do Brasil. Boletim do Museu de Biologia Mello Leitão, v. 34, p. 5-37, 2014.

Silva, T. C.; Silva, J. M.; Ramos, M. A. What factors guide the selection of medicinal plants in a local pharmacopoeia? A case study in a rural community from a historically transformed atlantic forest landscape. Evidence-Based Complementary and Alternative Medicine, v. 2018, 2018. https://doi.org/10.1155/2018/2519212

Soares, Z. A.; Lucena, R. F. P.; Ribeiro, J. E. S.; Carvalho, T. K. N.; Ribeiro, J. P. O.; Guerra, N. M.; Silva, N.; Pedrosa, K. M.; Coutinho, P. C.; Lucena, C. M.; Alves, C. A. B.; Sousa Júnior, S. P. Local botanical knowledge about useful species in a Semi-Arid Region from Northeastern Brazil. Gaia Scientia, v. 7, n. 1, p. 80-103, 2013.

Sousa, R. F.; Gomes, D. S.; Leite, A. P.; Santos, S. S., Alves, C. A. B.; Lucena, R. F. P. Estudo etnobotânico de Myracrodruon urundeuva Allemão em uma Comunidade Rural na Microrregião de Itaporanga, Paraíba, Brasil. Revista de Biologia e Farmácia, v. 7, n. 1, p. 72-83, 2012.

Souza, A. S.; Albuquerque, U. P.; Nascimento, A. L. B.; Santoro, F. R.; Torres-Avilez, W. M.; Lucena, R. F. P.; Monteiro, J. M. Temporal evaluation of the Conservation Priority Index for medicinal plants. Acta Botanica Brasilica, v. 31, n. 2, p. 169-179, 2017. https://doi.org/10.1590/0102-33062017abb0027

Sujarwo, W.; Caneva, G. Using quantitative indices to evaluate the cultural importance of food and nutraceutical plants: Comparative data from the Island of Bali (Indonesia). Journal of Cultural Heritage, v.18, p.342-348, 2016. https://doi.org/10.1016/ j.culher.2015.06.006

Tardío, J.; Pardo-de-Santayana, M. Cultural importance indices: A comparative analysis based on the useful wild plants of Southern Cantabria (Northern Spain). Economic Botany, v. 62, n. 1, p. 24-39, 2008. 
Tomazzoni, M. I.; Negrelle, R. R. B.; Centa, M. D. L. Fitoterapia popular: a busca instrumental enquanto prática terapêuta. Texto \& Contexto - Enfermagem, v. 15, n. 1, p. 115-121, 2006. https://doi.org/10.1590/S0104-07072006000100014

Trovão, D. M. B. M.; Freire, A. M.; Melo, J. I. M. Florística e fitossociologia do componente lenhoso da mata ciliar do Riacho de Bodocongó, Semiárido paraibano. Revista Caatinga, v. 23, n. 2, p. 78-86, 2010.

Vandebroek, I.; Reyes-García, V.; Albuquerque, U. P.; Bussmann, R.; Pieroni, A. Local knowledge: Who cares? Journal of Ethnobiology and Ethnomedicine, v. 7, n. 35, 2011. https://doi.org/10.1186/1746-4269-7-35

Velloso, A. L.; Sampaio, E. V. S. B.; Pareyn, F. G. C. Ecorregiões propostas para o bioma Caatinga. Recife: Associação Plantas do Nordeste; Instituto de Conservação Ambiental, The Nature Conservancy do Brasil, 2002.

Vitalini, S.; Iriti, M.; Puricelli, C.; Ciuchi, D.; Segale, A.; Fico, G. Traditional knowledge on medicinal and food plants used in Val San Giacomo (Sondrio, Italy): An alpine ethnobotanical study. Journal of Ethnopharmacology, v. 145, n. 2, p. 517-529, 2013. https://doi.org/10.1016/j.jep.2012.11.024

Yaseen, G.; Ahmad, M.; Sultana, S.; Alharrasi, A. S.; Hussain, J.; Zafar, M.; Shafiq-Ur-Rehman. Ethnobotany of medicinal plants in the Thar Desert (Sindh) of Pakistan. Journal of Ethnopharmacology, v. 163, p. 43-59, 2015. https://doi.org/10.1016/j.jep.2014.12.053

Informação da Licença: Este é um artigo Open Access distribuído sob os termos da Licença Creative Commons Attribution, que permite uso irrestrito, distribuição e reprodução em qualquer meio, desde que a obra original seja devidamente citada. 